\title{
LAS PRINCIPALES ETAPAS DE LA EVOLUCIÓN DEL METABOLISMO CELULAR. UNA APROXIMACIÓN EVOLUCIONISTA AL ESTUDIO DEL METABOLISMO
}

\author{
Chomin Cunchillos
}

Institut Charles Darwin International, París.

\section{RESUMEN}

Todos los fenómenos biológicos son el resultado de un proceso evolutivo. El metabolismo celular no es una excepción y en las células actuales se pueden encontrar huellas de ese proceso. En este trabajo ponemos de manifiesto, a través del análisis comparado, rasgos metabólicos suficientemente significativos como para permitirnos proponer un modelo filogenético de despliegue del metabolismo celular e identificar las principales etapas del mismo.

El primer modelo de este tipo fue desarrollado por F. Cordón (1990), en el contexto de su teoría de unidades de niveles de integración, y forma parte del desarrollo de la misma. El que presentamos aquí coincide plenamente con el de Cordón, pero está razonado a partir de argumentos que no son privativos de su teoría; con ello perseguimos independizar la discusión del primero de la argumentación de la segunda, de manera que la verosimilitud del modelo que proponemos, en el que se llega a las mismas conclusiones, refuerce los argumentos propios de la teoría y, como consecuencia, a ésta.

\section{SUMMARY}

All biological phenomena are the result of an evolutionary process. The cellular metabolism is not one exception and traces of this process can be found in modern cells. In this paper we are pointing out, through a comparative analysis, metabolic features meaningful enough as to allow us to propose a philogenetical pattern of a display of cellular metabolism and identify the main stages of it.

The first model of this type was developed by F. Cordón (1990) within his "Theory of Integration Levels Units" and it is part of the development of it. The pattern we are presenting here fully agrees whith that the Cordón's, but it is reasoned from arguments outside his theory which enables us to separate the model from the theory. Besides, to arrive at the same conclusions coming from different arguments reinforces the ones from that theory and, as a consequence, the theory itself. 


\section{INTRODUCCIÓN}

El metabolismo celular, como cualquier otro fenómeno biológico, es necesariamente el resultado de un proceso evolutivo. Este trabajo trata de mostrar, entre otras cosas, que dentro de la estructura de las células actuales se pueden encontrar datos que debidamente interpretados permiten reconstruir de forma verosímil su evolución.

El primer modelo de desarrollo evolutivo del metabolismo celular fue realizado por el biólogo español, recientemente fallecido, F. Cordón (1909-1999) en un contexto muy particular: como aplicación de su teoría de unidades de niveles de integración a la reconstrucción de la evolución biológica (Cordón, 1990; Cunchillos, 1997). En el trabajo que presentamos aquí se deduce la historia del metabolismo celular de consideraciones que no son privativas de esta teoría; con ello perseguimos independizar la discusión del modelo de la argumentación de la teoría; de manera que la verosimilitud del primero sirva como confirmación indirecta de la segunda (sin duda, el hecho de que, a partir de argumentos diferentes, se llegue al mismo modelo refuerza los utilizados por Cordón).

Dado que el desarrollo original del modelo que exponemos se hizo en el contexto de la teoría de unidades de niveles de integración, nos parece obligado comenzar por hacer una reflexión general sobre el trabajo de Cordón.

A lo largo del siglo XX, la ciencia, muy particularmente la biología, ha utilizado frecuentemente el concepto de nivel para justificar, mediante la «emergencia» de propiedades nuevas en la evolución, posturas antirreduccionistas (autonomistas desde el punto de vista de la biología) o, más concretamente, para defender el rechazo bien a la posibilidad bien a la pertinencia de algunas explicaciones reduccionistas [ver, por ejemplo, Bunge (1959), Rosenberg (1985) y Mayr (1988)]. Pero «el reduccionismo, si es posible, siempre es deseable» (Rosenberg, 1985), esto es, para descartar las explicaciones reduccionistas hay que argumentar de forma incontestable su imposibilidad teórica. Aplicando lo dicho al concepto de nivel, éste sólo podrá ser verdaderamente útil como argumento antirreduccionista, si se le dota del suficiente contenido teórico. A pesar de esto, han sido pocos los esfuerzos rigurosos por desarrollar este concepto. Cordón es una de las pocas excepciones, y su teoría de unidades de niveles de integración (a pesar de ser prácticamente desconocida) es considerada por algunos epistemólogos como «la más importante teorización actual de los niveles» y «el porvenir del pensamiento biológico moderno» (Tort, 1997) ${ }^{1}$.

1 Además de la teoría de unidades de niveles de integración de Cordón, el único esfuerzo teórico digno de señalar en este sentido es el desarrollo del concepto de nivel de complejidad hecho por v. Bertanffy (1949), muy limitado pero que no deja de ser el que utiliza generalmente la ciencia actual. En «Introduction à la théorie des niveaux d'intégration» Ch. Cunchillos (1997) se puede encontrar una discusión de las dos teorías. 
Esta teoría considera a las proteínas globulares como unidades de un nivel intermedio entre la molécula y la célula. Teniendo en cuenta que, en este marco, las unidades de nivel se corresponden con etapas de la evolución, en ésta, a partir del nivel molecular, se darían las siguientes:

$\rightarrow$ Evolución molecular $\rightarrow$ Evolución proteínica $\rightarrow$ Evolución celular $\rightarrow$

Los últimos veinticinco años Cordón los consagró esencialmente a aplicar su teoría a la reconstrucción del proceso de la evolución biológica, comenzando por la formación de las primeras proteínas en la evolución molecular (Cordón, 1977 y 1990). Su trabajo cubre hasta las primeras fases de la evolución celular. Uno de los resultados de este trabajo es su modelo de desarrollo filogenético del metabolismo (cuyo origen y desarrollo, según Cordón, habría tenido lugar en la etapa celular de la evolución). Este modelo, que constituye el primer modelo científico de evolución aplicada a este objeto, reconstruye el origen y la evolución del núcleo central del metabolismo celular formado por el ciclo del cítrico (o ciclo de Krebs) y las rutas de síntesis y de demolición de los aminoácidos, los ácidos grasos y los monosacáridos². El conjunto formado por estas rutas es común a todas las células actuales, procariotas y eucariotas, y en consecuencia, tuvo que desarrollarse en un proceso ocurrido en una etapa previa a su diversificación (en una célula ancestral de todas las actuales).

De su concepto de unidad de nivel de integración y del carácter de unidad de nivel directamente infracelular que, para él, tiene la proteína, Cordón (1994) concluye que la primera célula no pudo originarse sino a partir de una asociación heterotrofa de proteínas (Cordón, 1977) que se alimentaba de péptidos procedentes de restos de otras asociaciones, que le llegaban disueltos o en suspensión en el agua, y de los que extraía, uno a uno, los aminoácidos con los que, a continuación, cada proteína reconstruía su soma y la asociación, como consecuencia, el suyo propio. En este marco

2 No tenemos en cuenta, en este trabajo, el metabolismo de las moléculas complejas, ya que, aunque probablemente son los precursores tróficos de las que consideramos, su metabolismo se reduce, en todos los casos, a una hidrólisis que produce los monómeros correspondientes. La comparación de las diferentes hidrólisis no permite decidir sobre la anterioridad de una sobre otra, en cualquier caso, sea cual sea el polímero precursor, a partir de la hidrólisis se tuvo que desarrollar la ruta de demolición que corresponde a sus monómeros; como consecuencia, a partir del análisis comparado del metabolismo de estos se puede deducir el orden de utilización de las moléculas complejas de las que proceden.

Tampoco se consideran otros metabolitos como los aminoácidos aromáticos o las bases nitrogenadas, moléculas con alto contenido en nitrógeno que poseen una estructura aromática compleja. Estas moléculas muy probablemente han sido poco frecuentes en el medio en todos los momentos de la evolución, por lo que raramente formarían parte del ambiente trófico celular. En cualquier caso, no es verosímil que hayan sido utilizadas en el catabolismo primitivo por dos razones: 1/ su bajo contenido energético en comparación con las que consideramos (aminoácidos alifáticos, monosacáridos y ácidos grasos); y 2/ porque su proporción $\mathrm{C} / \mathrm{N}$ es poco adecuada a la composición celular. Además sus rutas metabólicas dependen de las de los otros monómeros para su ingreso y salida del ciclo, lo que sugiere que su despliegue filogenético fuese posterior. 
teórico, el metabolismo de la primera célula estaría reducido a la hidrólisis y la síntesis del enlace peptídico y a la utilización metabólica de los aminoácidos producidos en la primera; en consecuencia, las rutas metabólicas de estos tuvieron que ser las primeras en desarrollarse (antes que las de los monosacáridos y las de los ácidos grasos).

Como vemos más adelante, el análisis comparado de las rutas de síntesis y de demolición de los diferentes metabolitos nos lleva a la misma conclusión en cuanto a la anterioridad del metabolismo de los aminoácidos en el desarrollo filogenético. Ya hemos adelantado que para la argumentación de nuestra exposición no hemos utilizado los criterios característicos de la citada teoría, sino otros que se pueden asumir con carácter general, de forma que el modelo deducido de ellos, siendo evidentemente compatible, resulta independiente de la teoría de niveles de integración. Hay que señalar también que el análisis cladístico de las rutas de demolición de los aminoácidos alifáticos (único sector en que, de momento, se ha aplicado) ha confirmado las fases propuestas por el modelo que corresponden al desarrollo evolutivo del metabolismo de los aminoácidos (Cunchillos y Lecointre, 1999).

\section{EL MÉTODO DE TRABAJO EN LA RECONSTRUCCIÓN DEL DESARROLLO FILOGENÉTICO DEL METABOLISMO CELULAR}

En la reconstrucción de una filogenia, el análisis comparado se ha mostrado siempre como una herramienta eficaz; como vamos a ver, es también de gran ayuda para estudiar la evolución del metabolismo celular, siempre y cuando se escojan adecuadamente las características del metabolismo que van a ser comparadas.

La comparación es la herramienta ideal para reconstruir el pasado a partir de los datos del presente, nos permite identificar aquellos parecidos actuales que indican un origen común (homologías), establecer una subordinación de caracteres y, con ello, reconstruir una filogenia. La comparación, en el ámbito metabólico, entre células actuales pone en evidencia, sobre todo en bacterias pero también en células eucariontes, una gran variabilidad - diferentes formas de heterotrofismo, de autotrofismo fotosintético y quimiosintético, diversas formas de respiración o fermentación, etc. pero bajo esta diversidad se comprueba la existencia de un núcleo metabólico universal, común a todas las células actuales, formado por unas cincuenta rutas - entre otras, las de síntesis y las de demolición de los aminoácidos y los ácidos grasos, la glucolisis, la glucogénesis, la ruta de las pentosas y el ciclo de Krebs. Este núcleo es doblemente importante porque, por una parte, constituye la mayor parte del metabolismo de cualquier célula y, por otra, porque en él confluyen o de él parten las otras rutas más específicas. Esta universalidad fue detectada ya en 1926 por Kluyver, que enunció el concepto de «unidad de la bioquímica» considerado por Van Niel (1949) como el principal argumento a favor del origen monofilético de las células. Dicho de 
otra manera, ese núcleo común tuvo que desarrollarse durante una etapa inicial de la evolución celular, en una célula ancestral de todas las actuales ${ }^{3}$. Como consecuencia, las diferencias metabólicas que podemos observar hoy entre dos especies celulares (p. e. la presencia de una transformación o ruta metabólica diferente) se deben atribuir bien a adquisiciones posteriores al desarrollo del citado núcleo común, que nos informan sobre el desarrollo del metabolismo en una etapa posterior, o bien cabe la posibilidad de que tales diferencias representasen adquisiciones anteriores al despliegue de ese conjunto, conservadas en unas especies y perdidas en otras, en cuyo caso su comparación tampoco nos orienta sobre la etapa que queremos reconstruir.

A otro nivel, el de la estructura de las proteínas, la universalidad del citado núcleo metabólico supone que una misma transformación metabólica sea llevada a cabo, en diferentes especies, por enzimas que, teniendo la misma especificidad de función y de substrato, presentan entre sí importantes diferencias estructurales. Admitido el desarrollo durante una etapa común a la evolución de todas las células actuales del núcleo metabólico cuya historia tratamos de reconstruir, es obligado suponer que las proteínas que realizan la misma función enzimática en distintas especies celulares tienen un origen común, esto es, se han diferenciado a partir de una proteína ancestral y, por consiguiente, estas diferencias han aparecido después de la separación de esas especies. La comparación entre las estructuras de dichas proteínas puede servir para deducir su evolución a partir del momento de separación de dichas especies celulares, no así para reconstruir la historia del núcleo metabólico común, desarrollado en una etapa anterior. Los mismos argumentos nos permiten descartar, para los fines que nos proponemos, la comparación entre las secuencias de nucleótidos de los genes correspondientes a esas proteínas. Sin embargo esta conservación de la función obliga a pensar que las diferencias detectadas no han afectado a los rasgos estructurales de los centros activos que son necesarios para la catálisis (p. e. en la triosa-fosfatoisomerasa, se puede substituir, por otro aminoácido, cualquier histidina diferente a la his-95, que forma parte del centro activo, sin que se vea afectada la actividad catalítica del enzima, pero la substitución de este aminoácido por asparraguina supone una disminución de 10.000 veces de esta actividad, (Knowles 1991), de lo que es fácil deducir que esa histidina es uno de los rasgos estructurales necesarios para la catálisis y que habrá sido preservado en la evolución, si bien hay que tener en cuenta que el que se haya conservado su posición relativa en el centro activo - en la estructura terciaria de la proteína - no necesariamente quiere decir que se haya conservado su posición en la secuencia de aminoácidos - no necesariamente tiene que tratarse de la

3 La falta de sentido de las transformaciones metabólicas aisladas nos impone el hecho de que el metabolismo (considerado como un conjunto de transformaciones organizadas en rutas, lo que supone otro de proteínas funcionando coordinadamente) tiene que haberse desarrollado en la etapa celular de la evolución. (Probablemente haya existido un metabolismo precelular, correspondiendo a la actividad enzimática de proteínas aisladas no coordinadas en un conjunto organizado). 
posición 95). Parece, pues, que sería muy interesante poder comparar, de forma ordenada y sistemática, las estructuras de los centros activos de proteínas con parecidas y diferentes funciones enzimáticas, pertenecientes a la misma y a distintas especies celulares, con el fin de tratar de reconstruir la evolución de dichos centros a lo largo del desarrollo del metabolismo celular. Desgraciadamente, las dificultades para ello son todavía enormes y, hasta el momento, existen pocos datos en ese sentido. (Hay que destacar el excelente trabajo de revisión de la estructura del centro activo de la triosa-fosfato-isomerasa, realizado por Knowles, que acabamos de citar).

Dada la estricta complementariedad entre cada centro activo y la reacción que cataliza, una alternativa a la comparación de los primeros es la de las propias transformaciones metabólicas, con la ventaja añadida de que conocemos mucho mejor los mecanismos enzimáticos de las transformaciones metabólicas que la estructura de los correspondientes centros activos. Cordón (1990) se ha apoyado, en parte, en esta metodología para construir su modelo. Por otra parte, como señalan, entre otros, Zuckerkandl y Pauling (1965) las estructuras biológicas conservan inscrita en su organización gran parte de su propia historia. Como vemos a continuación, una reflexión sobre la estructura y la función del metabolismo celular permite obtener datos pertinentes a la reconstrucción de esa historia.

En nuestro intento de reconstruir el desarrollo evolutivo del metabolismo celular hemos partido, entre otras consideraciones, del análisis comparado: 1/ de las transformaciones metabólicas entre sí; 2/ de su organización en rutas; y 3/ de otros rasgos del metabolismo de interés filogenético. La comparación entre transformaciones metabólicas se ha mostrado particularmente útil para decidir el origen común de dos de ellas o la anterioridad evolutiva de una respecto a otra, lo que ha permitido a Cordón reconstruir con gran detalle el desarrollo evolutivo del metabolismo celular. Sin embargo, como vemos a continuación, para establecer las grandes etapas de este desarrollo (a las que, por razones de espacio, nos limitamos aquí), hay que recurrir a otro tipo de comparaciones.

\section{LAS PRINCIPALES ETAPAS DEL DESARROLLO DEL METABOLISMO CELULAR}

\section{El metabolismo de la primera célula}

El metabolismo de las células actuales es un complejo proceso constituido por un millar de reacciones químicas (catalizadas por proteínas globulares: los enzimas) ordenadas en un centenar de secuencias (las rutas metabólicas) que, en líneas generales, conectan la entrada de alimento, su aprovechamiento, la síntesis de componentes del soma celular, la de reservas y el aprovechamiento de éstas. Como cualquier otro fenómeno biológico, el metabolismo no puede ser sino un resultado de la evolución, 
su propia complejidad sugiere la de su historia evolutiva. Parece lógico pensar que el metabolismo adquirió esta complejidad en el curso de su evolución y que, por lo tanto, tuvo que consistir originariamente en un proceso mucho más simple.

La evolución biológica se interpreta generalmente como el resultado de la selección natural de variantes originadas por mutación -esto es, cualquier proceso evolutivo relativo a los seres vivos debe ser interpretado como el producto de la selección de variantes proteicas surgidas por mutación- lo que implica: 1/ la aparición de proteínas nuevas surgidas a partir de las anteriores, y $2 /$ la selección de las más adecuadas. Dado que el metabolismo celular es el producto de la actividad de numerosas proteínas (enzimas), debemos pensar que el millar largo de enzimas, organizados en unas cien rutas metabólicas, que constituyen la base somática del metabolismo de cualquier célula actual $-\mathrm{o}$, si se prefiere, de cuya actividad resulta su metabolismoprocede de un corto número de enzimas organizados en pocas rutas muy sencillas y que éstas se han ido alargando, y aumentado su número hasta la situación actual, por aparición de nuevos enzimas -a partir de la mutación de los anteriores y la selección de sus variantes más adecuadas.

La simplicidad del metabolismo de las primeras células no solo nos viene sugerida por el argumento anterior, sino que si consideramos la estructura y función de su substrato somático, los enzimas, concluimos lo mismo. La identidad de naturaleza (proteínas globulares) y de función (enzimática) de todos ellos hace obligado pensar que han tenido un origen común y, como consecuencia, que todos proceden de un primer enzima a partir del cual se fueron diferenciando los demás.

Así pues, parece evidente que el desarrollo evolutivo del metabolismo celular se acompañó del correspondiente proceso de diferenciación enzimática. Las primeras células poseerían un metabolismo muy sencillo, reducido a pocos enzimas, que, a lo largo de la evolución, se volvería cada vez más complejo por adquisición de nuevas transformaciones metabólicas (al diferenciarse nuevos enzimas a partir de los ya existentes) que alargarían progresivamente las rutas anteriores. La reconstrucción de la historia del metabolismo ha de consistir, pues, en identificar las rutas más primitivas, dentro de éstas las transformaciones o secuencias de transformaciones que se originaron primero, y, a continuación, el orden de aparición de las secuencias y rutas sucesivas.

\section{El orden de aparición de las transformaciones metabólicas}

Ya en 1945 Horowitz señaló que, dado que los metabolitos intermedios, en general, no tienen interés fisiológico y la capacidad para producirlos no confiere ninguna ventaja selectiva, las reacciones individuales solo tienen sentido para el organismo cuando se les considera en su conjunto, pero que carecen de él contempladas indivi- 
dualmente. Si se tiene en cuenta, también, que la aparición de una nueva transformación solo puede ser ventajosa si se integra en el metabolismo anterior y, además, mejora su eficacia (de otro modo el enzima correspondiente será eliminado), parece imponerse un orden de despliegue en el desarrollo de cada ruta metabólica.

A partir de esta consideración, Horowitz dedujo que el despliegue de las rutas de síntesis tuvo que seguir el orden inverso al que siguen los enzimas dentro de la ruta (el primero en aparecer sería el último de la ruta, el segundo el penúltimo y así sucesivamente) de forma que, obtenido el producto final de la ruta que le confiere su sentido fisiológico, el desarrollo de ésta estaría dirigido por la ventaja de obtenerlo a partir de precursores cada vez más sencillos.

Una argumentación semejante aplicada a las rutas de demolición nos lleva a la conclusión de que su desarrollo tuvo que seguir el mismo orden que presentan los enzimas en la ruta -la primera transformación estaría impuesta por el substrato de partida, procedente del alimento, y la aparición de las demás por la ventaja de intensificar su demolición.

Estas conclusiones parecen válidas para rutas de síntesis o de demolición cuyos productos finales o substratos iniciales, respectivamente, fueran impuestos a la célula desde el exterior por formar parte, al menos inicialmente, de su alimento -bien por ser constituyentes del soma tomados directamente del medio al comienzo, sintetizados metabólicamente después, bien por ser aprovechados como fuentes de energía a través de su demolición.

Es posible imaginar, sin embargo, otros escenarios alternativos para moléculas obtenidas por las propias células en el curso de su evolución por modificación de otras anteriores que fueran, hasta ese momento, productos finales o metabolitos intermedios de rutas ya existentes (cuyo origen podríamos denominar metabólico). Las nuevas secuencias de síntesis se desarrollarían progresivamente por adición de nuevos enzimas a rutas o secuencias anteriores (coincidiendo su orden de aparición con el de las transformaciones en la ruta: de precursor a producto). Las rutas de demolición, en este caso, se desplegarían conducidas por la ventaja de aprovechar los nuevos productos sintetizados (siguiendo el orden inverso al de las transformaciones en la ruta).

En resumen, se pueden diferenciar dos orígenes evolutivos diferentes para las moléculas metabólicas que condicionarían el orden de despliegue de sus respectivas rutas de demolición y de síntesis:

1. moléculas de origen trófico, cuyas rutas de demolición se desarrollarían en el sentido alimento $\longrightarrow$ residuos (siguiendo el mismo orden de las transformaciones en la ruta), mientras que las correspondientes a las síntesis seguirían el orden inverso producto final $\longrightarrow$ precursores.

2. moléculas de origen metabólico, para las que el despliegue ocurriría en el sentido contrario: de precursores a producto final, para las de síntesis (el mismo orden 
de las transformaciones en la ruta) y, en el caso de las de demolición, hacia la de moléculas de tamaño progresivamente mayor.

Hay que insistir, además, en que, en uno y otro caso, las presiones selectivas que dirigieron el despliegue tuvieron que ser diferentes: 1/ para las moléculas de origen trófico, intensificar la demolición (como medio para aumentar el rendimiento obtenido del alimento) en las rutas de demolición; 2/ conseguir sintetizar los productos finales a partir de precursores cada vez más sencillos, en las correspondientes de síntesis; 3/ sintetizar metabolitos de tamaño creciente (hasta uno óptimo) en los que se conserve una determinada estructura, en las rutas de síntesis de moléculas de origen metabólico; y 4/ aprovechar los productos de los nuevos tramos de síntesis, en las de demolición.

Así pues, parece claro que el tipo de despliegue que haya tenido una ruta habrá dependido del papel inicial de la correspondiente molécula dentro de la historia del metabolismo. Teniendo esto en cuenta, Cordón (1990) propone un desarrollo impuesto desde el exterior para las rutas de los aminoácidos (de productos a precursores para las rutas de síntesis, de alimento a residuos en las de demolición); y como moléculas sintetizadas de nuevo por la célula para los ácidos grasos y los monosacáridos (aumento progresivo del tamaño de los metabolitos en las rutas de síntesis y demolición de metabolitos cada vez mayores para las de demolición).

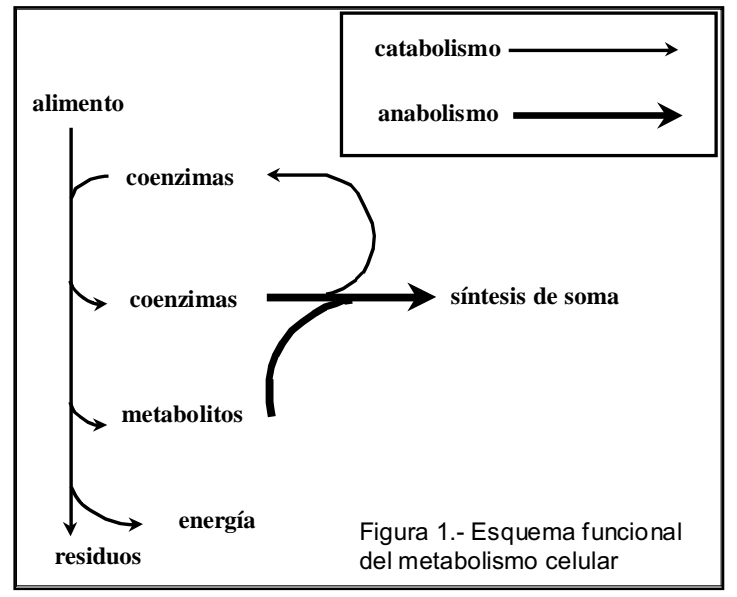

\section{La primera ruta}

Como hemos indicado, en este trabajo se trata de reconstruir las líneas generales de la evolución del núcleo metabólico formado por el ciclo del cítrico (o ciclo de Krebs) y 
las rutas de síntesis y de demolición de los aminoácidos, los ácidos grasos y los monosacáridos. En este conjunto de rutas que es común a las células actuales y tuvo que desarrollarse en una célula ancestral de todas ellas, se pueden distinguir tres sectores:

- el de los aminoácidos, formado por el ciclo del cítrico y las rutas de síntesis y demolición de todos los aminoácidos alifáticos;

- el de los ácidos grasos, que está formado por las rutas de síntesis y demolición de los ácidos grasos;

- el de los monosacáridos, que está formado por las rutas de síntesis (glucogénesis y ciclo de Calvin), de demolición (glucolisis) y de intertransformación (ruta de las pentosas) de los monosacáridos.

Cada ruta metabólica, a su vez, está constituida por un conjunto ordenado de transformaciones metabólicas, estructuradas somáticamente sobre proteínas con función enzimática (enzimas) que:

- en el caso de las rutas de demolición, transforman en residuos las moléculas (de aminoácidos, ácidos grasos o monosacáridos) procedentes del alimento o de las reservas celulares correspondientes;

- y en el caso de las rutas de síntesis, parten de metabolitos intermedios, suministrados por las rutas de demolición, para convertirlos en aminoácidos, en ácidos grasos o en monosacáridos que están destinados a las reservas correspondientes o a la alimentación de rutas de síntesis de moléculas más complejas.

Como resultado, las rutas de demolición quedan situadas entre la entrada de alimento y su utilización por la célula, proporcionando a ésta las moléculas y la energía necesarias para mantener su actividad (como se representa esquemáticamente en la Figura 1). Así, el catabolismo aprovisiona con piezas y coenzimas las rutas de síntesis, de tal manera que se intercala entre el alimento y su utilización en el anabolismo, y, como consecuencia, éste último depende totalmente de los metabolitos que le suministran las rutas de demolición. De esta situación se deduce la prioridad evolutiva de las primeras rutas de demolición respecto a las correspondientes de síntesis.

Parece, pues, evidente que el desarrollo filogenético del metabolismo tuvo que comenzar por el de una ruta dedicada a demoler moléculas procedentes del alimento celular. Pero ¿a cuál de los tres sectores considerados hay que asignar esta primera ruta?

\section{La primera ruta de demolición}

Si se acepta el supuesto de que la primera célula se originó a partir de una asociación de proteínas (hipótesis defendida por Cordón, y que, como hemos indicado, es 
una consecuencia del hecho de considerar la proteína globular como único nivel entre el molecular y el celular), el alimento de la primera célula tuvo que consistir necesariamente en péptidos procedentes de restos de asociaciones de proteínas, y de ello se deduce que sus primeras rutas metabólicas estuvieron dedicadas a la demolición de aminoácidos. Esta consecuencia, se nos dirá, no tiene este grado de evidencia más que si, dentro del contexto de la teoría de niveles, se admite de partida el carácter de nivel de integración directamente infracelular de la proteína; sin embargo, como vemos a continuación, el análisis comparado del metabolismo celular nos conduce a la misma conclusión (lo que constituye una confirmación indirecta del postulado de Cordón). Entre los argumentos que el estudio comparado del metabolismo nos aporta a favor de este supuesto merecen ser destacados los siguientes:

1. La composición química de las moléculas correspondientes a cada uno de los tres sectores es un dato que, a este respecto, parece decisivo. Las de los aminoácidos son las únicas que pueden suministrar los átomos necesarios tanto para sus síntesis como para las de los otros compuestos; como consecuencia, se puede entender el carácter de «vanguardia» de este sector en el desarrollo evolutivo, ya que, desde su origen, sus rutas de demolición y de síntesis pudieron constituir un núcleo metabólico incipiente pero con pleno sentido funcional (la demolición del alimento suministraría a la célula la energía suficiente para mantener su actividad, y las piezas para reconstruir su soma), a partir del cual pudieron desplegarse los otros dos sectores, comenzando por sus respectivas rutas de síntesis. No sería posible, sin embargo, deducir un modelo coherente de desarrollo del metabolismo ni a partir del de los monosacáridos ni del de los ácidos grasos.

2. La comparación entre las estructuras químicas de estas moléculas apoya igualmente esta interpretación. Mientras que los ácidos grasos y los monosacáridos tienen una estructura monótona, en la que solo varía el número de carbonos de la cadena, las correspondientes a cada uno de los diferentes aminoácidos metabólicos únicamente tienen en común el extremo amino-ácido $\left(\mathrm{COOH}-\mathrm{CH}-\mathrm{NH}_{2}\right)$, siendo el resto de la cadena exclusivo de cada uno de ellos (pudiendo incluir $\mathrm{N}$ y $\mathrm{S}$, además de átomos de $\mathrm{C}, \mathrm{H} \mathrm{y} \mathrm{O}$ ), sin que exista la menor regla en su estructura (Figura 2).

\begin{tabular}{|lll|}
\hline Aminoácidos & $\begin{array}{c}\mathrm{COOH}-\mathrm{CH}-\mathrm{R} \\
\mathrm{NH}_{2}\end{array}$ & (R es característico de cada aminoácido) \\
Ácidos grasos & $\mathrm{COOH}-\left(\mathrm{CH}_{2}\right)_{\mathrm{n}}-\mathrm{CH}_{3}$ \\
Monosacáridos (aldosas) & $\mathrm{CH}_{2} \mathrm{OH}-(\mathrm{HCOH})_{\mathrm{n}}-\mathrm{COH}$ \\
Monosacáridos (cetosas) & $\mathrm{CH}_{2} \mathrm{OH}-(\mathrm{HCOH})_{\mathrm{n}}-\mathrm{CO}-\mathrm{CH}_{2} \mathrm{OH}$ \\
Figura 2.- Fórmulas generales de los aminoácidos, ácidos grasos y monosacáridos \\
\hline
\end{tabular}


La diversidad estructural de los aminoácidos sería la de esperar para moléculas cuya utilización en el metabolismo tenga un origen trófico, esto es, que, por formar parte de su ambiente trófico, han sido impuestas a la célula desde el exterior y a las que ésta se ha visto forzada a adaptarse. Mientras que la homogeneidad de los monosacáridos y los ácidos grasos se explica bien si suponemos para ellas un origen metabólico, como moléculas sintetizadas por la célula a partir de metabolitos intermedios de alguna ruta desarrollada antes, cuyos rasgos estructurales, por resultar adecuados a una función nueva, se conservan con modificaciones de tamaño.

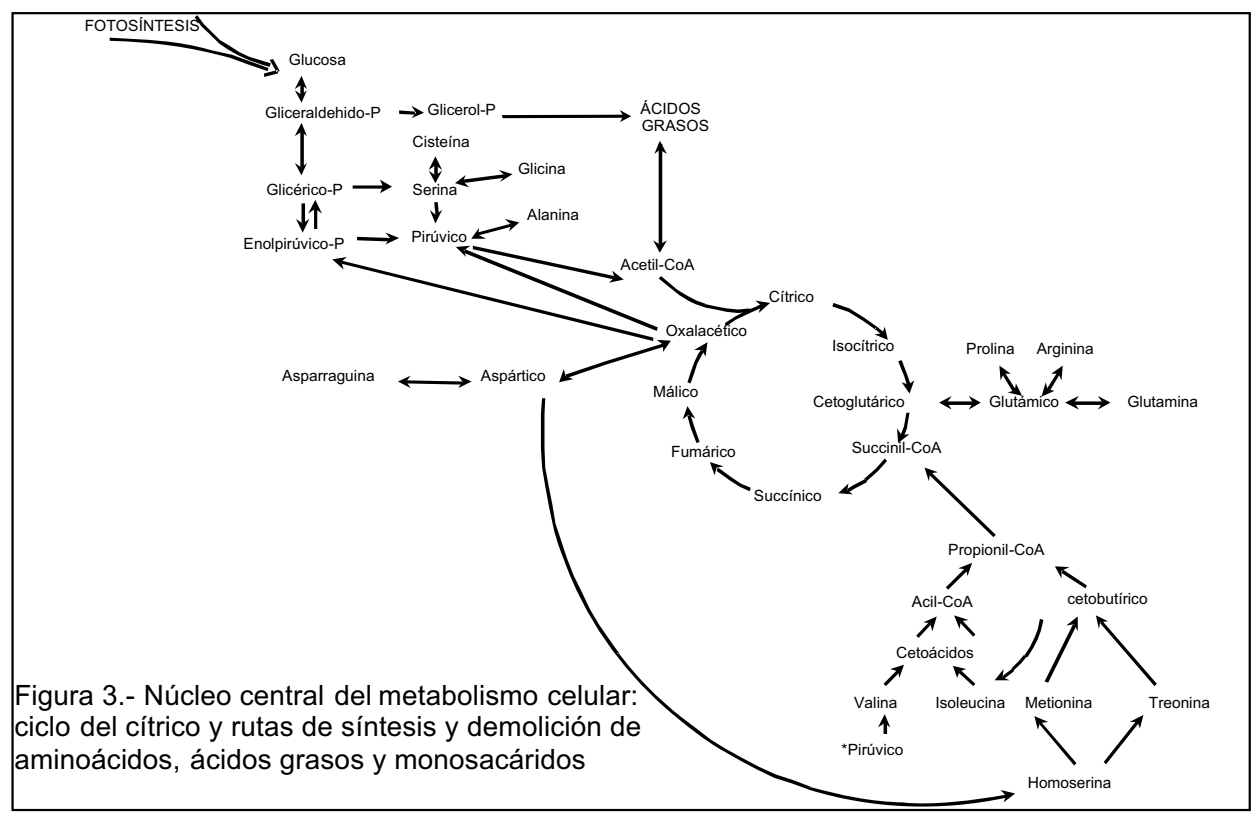

3. Si nuestra interpretación anterior es acertada (origen trófico para los aminoácidos metabólicos y metabólico para los primeros monosacáridos y ácidos grasos), tuvieron que ser diferentes las presiones selectivas que guiaron el desarrollo evolutivo del metabolismo en uno y otro caso, lo que debería haber quedado reflejado en la estructura actual de su metabolismo. Como vemos a continuación, la estructura de las rutas metabólicas de los tres sectores es coherente con esta interpretación.

El sector de los aminoácidos posee una estructura muy compleja, en la que:

- para cada aminoácido existe una ruta de síntesis y otra de demolición que, aunque compartan algún tramo con otras, siempre poseen la exclusividad de alguno propio; 
- hay un gran número de tipos de transformaciones metabólicas diferentes;

- en sus rutas metabólicas se recurre a muchos coenzimas diferentes (diez en total);

- las rutas son muy complejas, estando formadas por un número variable, de una a otra, de tramos metabólicos diferentes;

- algunas rutas de demolición terminan paradójicamente en un tramo de síntesis;

— las rutas de demolición y las rutas de síntesis no presentan casi ningún paralelismo.

Por el contrario, los otros dos sectores presentan una estructura mucho más simple, donde:

- hay pocas rutas por sector;

— éstas están formadas por un número pequeño de transformaciones diferentes;

- en ellas se recurre a pocos coenzimas diferentes (tres en cada sector);

- las rutas de síntesis y de demolición son rigurosamente inversas;

- casi siempre, las transformaciones metabólicas están ordenadas en tramos cortos repetidos de manera monótona a lo largo de las rutas.

La complejidad del sector de los aminoácidos parece reflejar un desarrollo forzado por la estructura de los metabolitos, como si ésta, de acuerdo con el supuesto precedente, estuviera impuesta desde el exterior por la composición del alimento celular. La adaptación de la célula a una explotación mejor de su ambiente trófico impulsaría el desarrollo de nuevas transformaciones de demolición capaces de obtener del alimento un mayor rendimiento metabólico. La diversidad estructural de los aminoácidos alimentarios justificaría la variedad de soluciones (transformaciones, coenzimas y tramos de síntesis en rutas de demolición) a las que se recurre en este sector.

Por su parte, la sencillez de las rutas metabólicas de los otros dos sectores sugiere un desarrollo dirigido a la obtención de metabolitos de tamaño creciente, mediante la repetición de tramos de alargamiento en sus rutas de síntesis, conservando unos rasgos estructurales (sin duda por ser los adecuados a una determinada función - probablemente, al comienzo por lo menos, la de reserva), que se habrían obtenido inicialmente en un metabolito pequeño en el curso anterior de la evolución. Las rutas de demolición se habrían desplegado, invirtiendo rigurosamente las de síntesis, dirigidas por la ventaja selectiva de aprovechar metabólicamente los productos obtenidos en cada nuevo tramo de síntesis.

4. El que una de las funciones principales de los ácidos grasos y de los monosacáridos sea la de reserva celular, refuerza igualmente los argumentos anteriores.

5. Finalmente, las rutas de síntesis y demolición de los aminoácidos guardan con el ciclo unas relaciones más completas que las de los monosacáridos y las de los ácidos grasos. Como se representa en la Figura 3, las cuatro entradas del ciclo del 
cítrico (oxalacético, $\alpha$-cetoglutárico, acetil-CoA y succinil-CoA) son utilizadas por las rutas de demolición de los aminoácidos (como vemos más adelante estas entradas permiten hacer una clasificación filogenética de los mismos), siendo tres de ellas exclusivas de este sector, mientras que la tercera, el acetil-CoA, es utilizada, además, por los otros dos. Algo parecido ocurre con las dos salidas del ciclo (oxalacético y $\alpha$ cetoglutárico), ambas alimentan rutas de síntesis de aminoácidos, pero solo una, el oxalacético, es utilizada por los otros dos sectores.

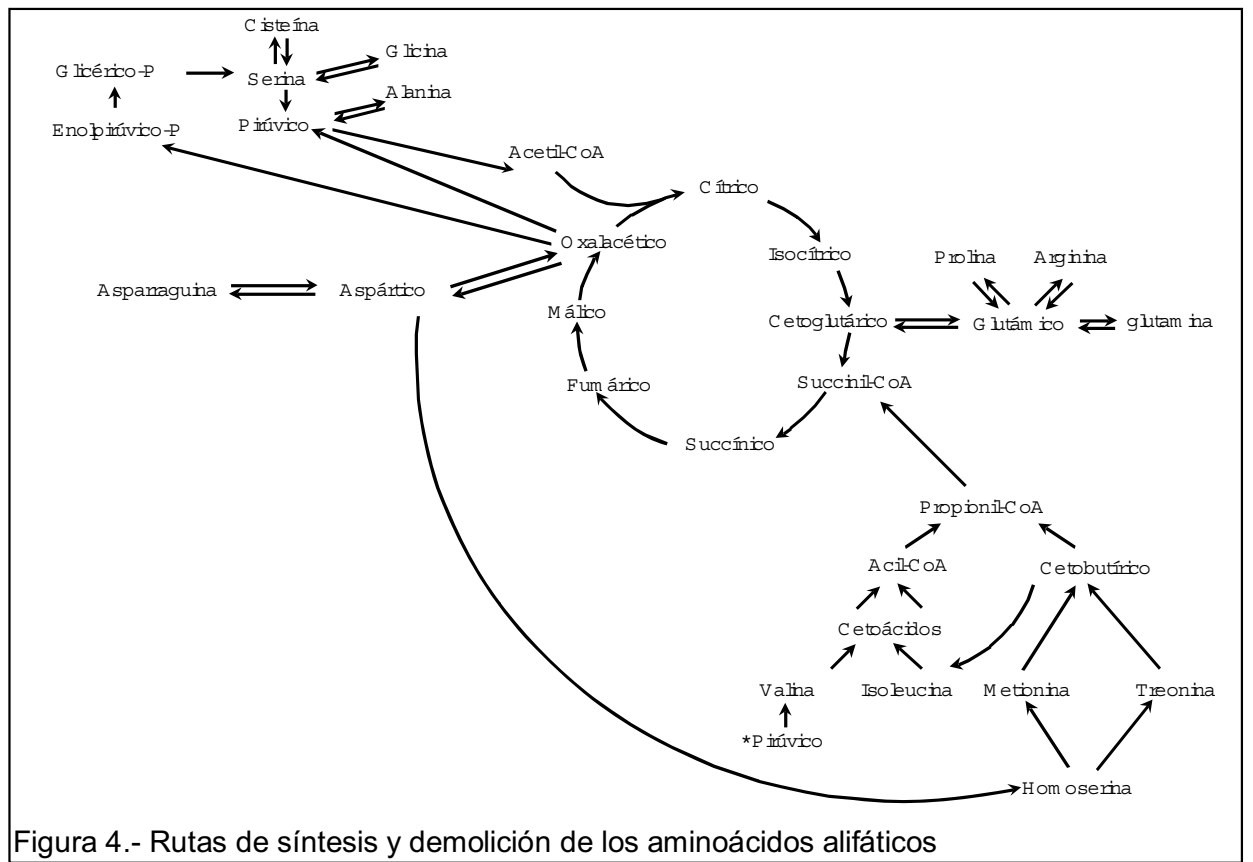

Como resultado, el conjunto formado por el ciclo del cítrico y las rutas de demolición y de síntesis de los aminoácidos (Figura 4) puede interpretarse como un núcleo unitario, con pleno sentido para las síntesis y las demoliciones, al que se han adosado posteriormente los otros dos sectores. En apoyo de esta interpretación, el ciclo del cítrico propiamente dicho consiste en la interconversión de dos $\alpha$-cetoácidos, el oxalacético y el $\alpha$-cetoglutárico, productos de la desaminación de dos aminoácidos, el aspártico y el glutámico, respectivamente.

Los argumentos precedentes parecen suficientes para defender la hipótesis de que la primera ruta metabólica en desplegarse fue una de demolición de algún aminoácido de origen alimentario, y que esta ruta tuvo que desplegarse progresivamente en el 
sentido aminoácido $\longrightarrow$ ciclo del cítrico (el mismo de la actuación de los enzimas en la ruta). Lo que nos permite postular dos grandes fases en el desarrollo del metabolismo celular: durante la primera habría tenido lugar el despliegue progresivo de las rutas de demolición y de síntesis de los aminoácidos que culminaría en la formación del ciclo del cítrico (en el curso de esta fase la intensificación de las demoliciones habría sido la principal ventaja selectiva), lo que daría lugar a un primer núcleo metabólico a partir del cual, en una segunda fase, se desarrollarían los otros dos sectores mediante el despliegue de transformaciones simples (muchas de ellas presentes en el metabolismo de los aminoácidos), estructuradas en tramos dobles de síntesis y demolición inversos, repetidos regularmente.

Así pues, la hipótesis según la cual el primer sector metabólico en desarrollarse fue el de los aminoácidos y que tal desarrollo comenzó por el de una de sus rutas de demolición, postulado obligado en la perspectiva de la teoría de las unidades de niveles de integración, se deduce también del análisis comparado de los tres sectores (lo que, como hemos indicado, confirma indirectamente dicho postulado).

\section{Las rutas de demolición de los aminoácidos}

A continuación, consideramos el sector de los aminoácidos alifáticos, más concretamente sus rutas de demolición, con el fin de poner de manifiesto algunos rasgos de las mismas que, en nuestra opinión, son suficientemente significativos, desde un punto de vista filogenético, como para permitirnos avanzar un modelo de despliegue evolutivo de este sector e identificar las principales etapas del mismo. Vamos a considerar tres aspectos de estas rutas:

— los tramos metabólicos que comparten;

- su estructura en tramos funcionalmente diferentes;

- y el recurso a coenzimas en ellas.

En la Figura 4, que representa la estructura general de este sector, incluido el ciclo del cítrico, podemos observar que, aunque hay tantas rutas de síntesis y de demolición como aminoácidos, cada una de ellas no es enteramente exclusiva de un único aminoácido, sino que siempre posee al menos un tramo metabólico en común con otras. Estos tramos, que, como se discute a continuación, pueden representar homologías, por lo que son una de las características con significado evolutivo más evidente, dan lugar a un ordenamiento en red de las rutas, que confluyen (las de demolición) en determinados metabolitos para compartir a continuación tramos metabólicos comunes, o se separan (las de síntesis) después de un tramo común en otros independientes. Ahora bien, estos tramos compartidos solo admiten dos explicaciones evolutivas: 
- o bien tienen un origen múltiple, aparecieron independientemente en cada una de las rutas que los comparten, y son, por tanto, tramos análogos;

- o bien tienen un origen común, primero se originaron en una ruta y a continuación se extendió su aplicación a otras, en cuyo caso se trata de tramos homólogos 4 .

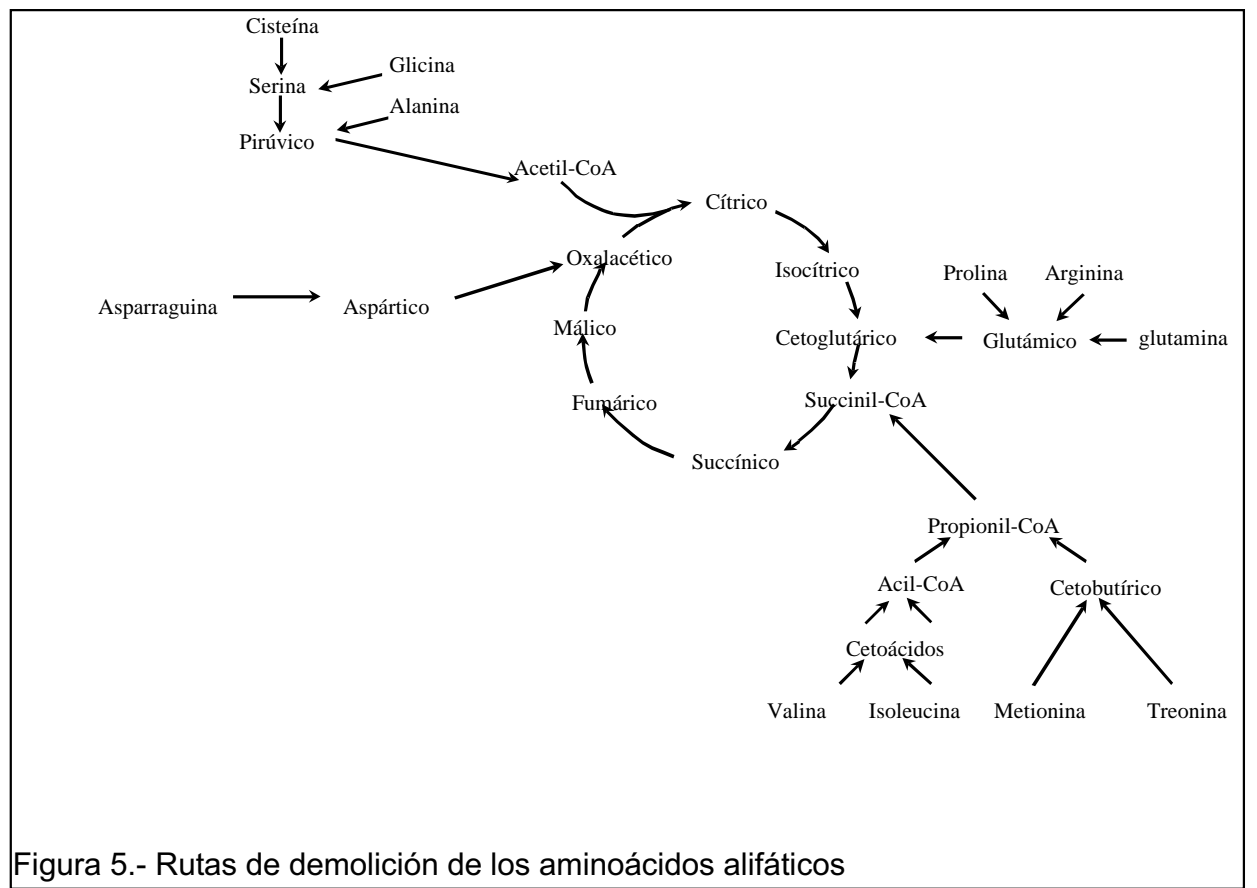

En la Figura 5 se representan solo las rutas de demolición de los aminoácidos alifáticos ${ }^{5}$. Se puede observar como éstas confluyen progresivamente hacia el ciclo en

4 Para decidir si un determinado parecido representa una analogía o una homología, es preciso evaluar el grado de este parecido. En nuestro caso, cuanto mayor sea la complejidad de la transformación metabólica o del tramo de ruta comunes, mayor será nuestra convicción de que corresponden a una homología, y, en consecuencia, que revelan un origen común.

5 Por razones de simplicidad en la representación, en las Figuras 3, 4 y 5 faltan las rutas de la leucina y la lisina que entran en el ciclo a través de acetil-CoA pero de forma diferente a como lo hacen los aminoácidoss del grupo 3. El análisis de sus transformaciones metabólicas muestra que guardan relaciones evolutivas con las de la isoleucina y la valina, en el caso de la leucina, y con el cierre del ciclo, la lisina (Cordón, 1990). 
el que desembocan a través de cuatro entradas (oxalacético, $\alpha$-cetoglutárico, acetilCoA y succinil-CoA). Como consecuencia de esta confluencia, los tramos más próximos al ciclo son compartidos por un mayor número de rutas. Las rutas que poseen tramos comunes pueden reunirse en cuatro grupos, que corresponden a las cuatro entradas en el ciclo:

- las rutas del aspártico y la asparraguina, grupo 1, entran en el ciclo a través del tramo aspártico $\longrightarrow$ oxalacético;

- las rutas de demolición del glutámico, la glutamina, la prolina y la arginina, grupo 2, comparten el tramo glutámico $\longrightarrow \alpha$-cetoglutárico;

- las de la alanina, la serina, la cisteína y la glicina, grupo 3, confluyen en el tramo pirúvico $\longrightarrow$ acetil-CoA;

- finalmente, el grupo 4 está formado por las rutas de demolición de la metionina, la treonina, la valina y la isoleucina que tienen en común el tramo propionil-CoA $\longrightarrow$ succinil-CoA.

Como hemos indicado, las rutas de cada grupo comparten al menos un tramo, pero pueden tener en común más de uno - por ejemplo, las del grupo 4 poseen en común el tramo final, propionil-CoA $\longrightarrow$ succinil-CoA, pero dos de ellas, las de la metionina y la treonina, comparten además el tramo: $\alpha$-cetobutírico $\longrightarrow$ propionilCoA. Como los tramos compartidos pueden representar homologías, es de esperar que las rutas de cada grupo mantengan entre sí relaciones filogenéticas más estrechas que con el resto.

Dentro de las rutas de demolición podemos diferenciar tres tipos de tramos metabólicos desde el punto de vista de su funcionalidad para el catabolismo: 1/ los dedicados a la desaminación; 2/ los que conducen a la descarboxilación; y 3/ tramos de síntesis (situados, paradójicamente, en rutas de demolición). Si consideramos el tipo de tramos que componen cada ruta, éstas se pueden clasificar en tres tipos:

- las rutas de los grupos 1 y 2 están formadas solo por tramos de desaminación;

- las que corresponden al grupo 3 poseen un primer tramo de desaminación seguido de un segundo de descarboxilación;

— en el grupo 4, las rutas de demolición incluyen, además, un tercer tramo de carboxilación.

Esta correspondencia entre el agrupamiento de rutas (de acuerdo a su confluencia en el ciclo) y su tipología (de acuerdo al significado funcional de sus tramos constituyentes) - que es aun más patente cuando se tiene en cuenta el análisis comparado de las transformaciones metabólicas (Cordón, 1990) - apoya la hipótesis, que hemos adelantado, de una historia común para las rutas de demolición de cada grupo. Pero, además, nos permite hacer consideraciones transversales que permiten relacionar 
entre sí los desarrollos evolutivos de las rutas correspondientes a los diferentes grupos. Así, tenemos que:

- todas las rutas comienzan con un tramo de desaminación que produce $\alpha$ cetoácidos (las rutas de los grupos 1 y 2 se reducen a este tipo de tramos);

- las rutas de los grupos 3 y 4 añaden un segundo tramo dedicado a la descarboxilación del $\alpha$-cetoácido producido en el anterior (las rutas del grupo 3 están constituidas por estos dos tramos, entrando en el ciclo a través del acetil-CoA producido en la descarboxilación del pirúvico);

- y, finalmente, las rutas del grupo 4 contienen un tercer tramo de síntesis que convierte en succinil-CoA el propionil-CoA producido en los tramos de descarboxilación.

La generalidad de las desaminaciones respecto a las descarboxilaciones, y de éstas respecto a los tramos de síntesis, sugiere nuevamente que el orden de despliegue de la ruta fuera el esperado para una de demolición cuyo substrato venga impuesto desde el exterior por la composición del alimento (como hemos supuesto que tuvo que ocurrir con las rutas de demolición de los aminoácidos alifáticos), lo que resulta coherente con que los tramos finales de las rutas del grupo 4 sean de síntesis, aunque situados en el interior de rutas de demolición, lo que parece reflejar una complicación secundaria de la ruta correspondiente - como si su desarrollo hubiera estado condicionado por la evolución anterior del resto del metabolismo.

Todo ello induce a pensar que el desarrollo de las rutas de demolición de los aminoácidos tuvo lugar según la siguiente secuencia de acontecimientos: 1/ despliegue de las desaminaciones; $2 /$ despliegue de las descarboxilaciones; $3 /$ despliegue de los tramos de carboxilación del propionil-CoA en las rutas del grupo 4. Lo que implica, a su vez, que las primeras rutas en alcanzar su estado definitivo fueron las de los grupos $1 \mathrm{y}$ 2, a continuación se completarían las del grupo 3, y, finalmente, las del grupo 4.

$\mathrm{Si}$, como hemos supuesto, el desarrollo de las rutas de demolición de los aminoácidos tuvo lugar en el sentido aminoácido-substrato $\longrightarrow$ ciclo del cítrico (esto es, en el sentido de los enzimas en la ruta), puesto que en este sentido las rutas convergen progresivamente (Figura 5), su desarrollo evolutivo tuvo que ir acompañado de una confluencia cada vez mayor cuyo resultado final sería la formación del ciclo.

A continuación vamos a considerar otro aspecto del metabolismo de los aminoácidos que permite matizar el análisis anterior: se trata de la utilización de coenzimas en sus rutas de demolición. Desde un punto de vista filogenético parece lógico suponer que el recurso a coenzimas en la evolución del metabolismo fuera progresivo, aumentando su número con el tiempo, paralelamente al número y a la dificultad de las transformaciones metabólicas desarrolladas. En consecuencia, sería de esperar que las rutas de demolición de los aminoácidos pudieran ordenarse por el número de coenzimas utilizados en ellas. Confirmando este supuesto, en la Figura 6, los ami- 
noácidos aparecen ordenados según el número de coenzimas utilizados en sus rutas de demolición (creciente hacia la derecha) .

\begin{tabular}{|c|c|c|c|c|c|c|c|c|c|c|c|c|c|c|c|c|}
\hline \multirow{2}{*}{\multicolumn{2}{|c|}{$\begin{array}{l}\text { Coenzimas } \\
\text { utilizados }\end{array}$}} & \multicolumn{15}{|c|}{ Rutas de demolición de los aminoácidos } \\
\hline & & ASP & GLU & ASN & GLN & ARG & PRO & ALA & SER & CIS & GLI & MET & TRE & ILE & VAL LEU & LIS \\
\hline \multicolumn{17}{|c|}{ NAD (NADP) } \\
\hline \multicolumn{17}{|c|}{ Piridoxal-fosfato } \\
\hline \multicolumn{17}{|c|}{ FAD (FMN) } \\
\hline \multicolumn{17}{|c|}{ Tiamin-pirofosfato } \\
\hline \multicolumn{17}{|c|}{ Lipoamida } \\
\hline \multicolumn{17}{|c|}{ Coenzima A } \\
\hline \multicolumn{17}{|l|}{ АTP } \\
\hline \multicolumn{17}{|l|}{ Biotina } \\
\hline \multicolumn{17}{|c|}{ Cobalamina } \\
\hline \multicolumn{17}{|c|}{ Tetrahidrofólico } \\
\hline \multirow{4}{*}{$\begin{array}{l}\text { Entrada } \\
\text { en el } \\
\text { ciclo }\end{array}$} & Directa & & & & & & & & & & & & & & & \\
\hline & \begin{tabular}{|l|} 
Pirúvico \\
\end{tabular} & & & & & & & & & & & & & & & \\
\hline & Succinil-coA & & & & & & & & & & & & & & X & \\
\hline & Acetil-coA & & & & & & & & & & & & & & & \\
\hline $\begin{array}{l}\text { Figura } 6 .- \\
\text { GLU }=\text { glut } \\
\text { SER=seri } \\
\text { LEU =leuc }\end{array}$ & $\begin{array}{l}\text { Recurso a } \\
\text { támico, } \\
\text { na, CIS =cis } \\
\text { cina, LIS=lis }\end{array}$ & & & & & & & ió & & & $\begin{array}{l}\text { minoác } \\
\text { ina, } \\
\text { nina }\end{array}$ & & & & $\begin{array}{l}\mathrm{ASP}=\mathrm{aspá} \\
\mathrm{ALA}=\mathrm{al}\end{array}$ & \\
\hline
\end{tabular}

En primer lugar, hay que resaltar que el simple hecho de que este criterio permita ordenar los aminoácidos, como lo muestra la figura, supone necesariamente la existencia de algún tipo de relación (la que se postula u otra equivalente) entre los aminoácidos de una parte, y de la otra el número y el tipo de coenzimas utilizados en sus

6 La situación, aparentemente desordenada, de las rutas de la glicina y de la metionina en la figura 6 se explica por la estructura de estos aminoácidos. La glicina es el aminoácido más sencillo y su demolición comienza por una transformación de síntesis (en la que se utiliza el tetrahidrofólico) que permite que su ruta confluya con las de los demás aminoácidos del grupo 3. En cuanto a la metionina, su peculiar estructura (con un grupo metilo distal unido por un enlace tiol al resto de la molécula) justifica el recurso a tetrahidrofólico con el fin de aprovechar el grupo $\mathrm{CH}_{3}$ distal coordinando su metabolismo con el de la glicina. 
rutas de demolición. Si, como suponemos, el orden de las rutas de demolición de los aminoácidos en la figura está en relación con el de su desarrollo evolutivo sería de esperar, en una primera aproximación, que el desarrollo evolutivo definitivo de las rutas de demolición de los aminoácidos situados más a la izquierda en la figura (en las que se recurre a un menor número de coenzimas) se alcanzase antes.

En segundo lugar, el orden de los aminoácidos no es cualquiera, sino que, como se puede observar en la parte inferior de la Figura 6, se corresponde con las diferentes entradas de sus rutas de demolición en el ciclo del cítrico. De manera que, de la consideración de esta tabla, se puede deducir la misma clasificación de las rutas de demolición de los aminoácidos alifáticos que se ha deducido antes a partir de la consideración de los tramos compartidos por las mismas. (Los grupos 1 y 2 aparecen mezclados por razones evolutivas en las que no entramos aquí, pero que un análisis más detallado pone de manifiesto.)

Estos hechos sugieren, de nuevo, la precedencia evolutiva de las rutas de demolición correspondientes a los dos primeros grupos de aminoácidos (en las que se utiliza menor número de coenzimas).

Finalmente, si los criterios utilizados son válidos, el orden de las rutas de los aminoácidos, horizontalmente, debería corresponder, en vertical, con la utilización progresiva de coenzimas, y debería reflejar el orden de aparición de las transformaciones en las que estos son utilizados. De este modo se pueden agrupar y ordenar las transformaciones en cuatro tipos que debemos suponer sucesivos en la evolución:

1. las desaminaciones, con recurso a $\mathrm{NAD}^{+}$;

2. las transaminaciones, en las que se utiliza piridoxal-fosfato;

3. las descarboxilaciones, en las que se recurre a cuatro nuevos coenzimas: lipoamida, tiamin-pirofosfato, FAD y coenzima A;

4. y, finalmente, las metilaciones, en las que se utiliza tetrahidrofólico, y las carboxilaciones, en las que se recurre a ATP, biotina y cobalamina.

Se puede observar que el orden deducido a partir de este criterio coincide con el obtenido de la consideración de la funcionalidad de los tramos metabólicos y, nuevamente, con el que se supone para moléculas de origen trófico. Lo mismo ocurre con el agrupamiento de aminoácidos, al que se llega también desde la consideración de los tramos compartidos y que es coherente con la tipología de rutas hecha de acuerdo con su composición en tramos funcionales.

Hay que destacar que los tres criterios utilizados en el análisis de las rutas de demolición de los aminoácidos alifáticos (tramos compartidos -tomados como índices de posibles homologías-, funcionalidad de los tramos metabólicos y recurso a coenzimas) representan aspectos del metabolismo entre los que a priori no es fácil establecer ninguna relación, salvo, como hemos supuesto, la de que todos son el resultado de un mismo proceso evolutivo. El que a partir de tres criterios aparentemente independientes 
se llegue a las mismas consideraciones finales indica que entre esos tres aspectos del metabolismo existe una relación ¿qué otra puede ser que la filogenética?

En nuestra opinión, el análisis anterior permite defender dos conclusiones:

1. Un agrupamiento filogenético de las rutas de demolición de los aminoácidos:

- El grupo 1 contiene solo transformaciones de desaminación, utiliza únicamente un coenzima (NAD) y comparte el tramo aspártico $\longrightarrow$ oxalacético.

- El grupo 2 también contiene únicamente transformaciones de desaminación y utiliza un solo coenzima (NAD), sus rutas comparten el tramo glutámico $\longrightarrow \alpha$-cetoglutárico.

- El grupo 3 tiene un tramo de desaminación y otro de descarboxilación, utiliza seis coenzimas (NAD, piridoxal-fosfato, FAD, lipoamida, tiaminpirofosfato y coenzima A) y comparte el tramo pirúvico $\longrightarrow$ acetil-CoA.

- El grupo 4 contiene tres tramos funcionales diferentes (sucesivamente: de desaminación, de descarboxilación y de carboxilación), utiliza nueve coenzimas (los señalados para el grupo 3 más ATP, cobalamina y biotina) y comparte el tramo propionil-CoA $\longrightarrow$ succinil-CoA.

2. Un modelo de despliegue evolutivo de las rutas de demolición de los aminoácidos en el que se pueden distinguir las siguientes etapas:

- Primera etapa, que corresponde al desarrollo de las desaminaciones, al final de ella se alcanzaría el desarrollo definitivo de las rutas de síntesis y de demolición de los aminoácidos de los grupos 1 y 2 , y se habrían desarrollado además los tramos de desaminación en las rutas de demolición de los aminoácidos de los grupos 3 y 4; el análisis del recurso a coenzimas sugiere, como hemos visto, que en esta primera etapa se pueden diferenciar dos subetapas sucesivas, en la primera se desplegarían las desaminaciones con NAD y en la segunda las transaminaciones y las desaminaciones con recurso a piridoxal-fosfato.

- Segunda etapa, durante la cual se desarrollarían las descarboxilaciones en las rutas de demolición de los aminoácidos de los grupos 3 y 4, completándose con ello el desarrollo de las rutas de demolición del grupo 3 .

- Tercera y última etapa, a lo largo de la cual se desarrollarían las transformaciones que permiten la entrada en el ciclo del cítrico de los productos metabólicos de las descarboxilaciones en las rutas de demolición de los aminoácidos del grupo 4.

De acuerdo con este orden de desarrollo, se puede observar en la Figura 5 como dos de las cuatro entradas en el ciclo tienen lugar directamente a través de cetoácidos: oxalacético y $\alpha$-cetoglutárico, que son los productos de la desaminación de los 
aminoácidos aspártico y glutámico; mientras que las otras dos entradas se efectúan a través de metabolitos que han sufrido una transformación más intensa. La proximidad de los grupos 1 y 2 al ciclo, y el hecho de que éste último consista en dos rutas de conversión de un cetoácido en otro, son dos datos que sugieren que el desarrollo del ciclo pudo haber estado estrechamente unido con el del metabolismo de los aminoácidos y particularmente, al menos al comienzo, con el de los grupos 1 y 2 .

\section{PRINCIPALES PERÍODOS DEL DESARROLLO DEL METABOLISMO CELULAR}

Los argumentos anteriores nos parecen suficientes para defender un primer esquema de desarrollo del metabolismo celular. A grandes rasgos, se pueden distinguir dos fases: la primera que corresponde al desarrollo del metabolismo de los aminoácidos alifáticos, y que culminaría en la formación del ciclo del cítrico; y la segunda en la que tendría lugar el desarrollo del metabolismo de los ácidos grasos y de los monosacáridos.

La primera de estas dos grandes fases se puede dividir, a su vez, en tres etapas sucesivas:

- La Primera etapa corresponde al desarrollo de las desaminaciones y de las transaminaciones, con recurso a NAD y a piridoxal-fosfato como coenzimas, y en ella se alcanzaría la estructura definitiva del metabolismo de los aminoácidos de los grupos 1 y 2 . En esta etapa se pueden distinguir dos subetapas: durante la primera se utilizaría solo NAD y se desplegarían las desaminaciones en que se usa, en la segunda se recurriría, además, a piridoxal-fosfato y se desarrollarían las transaminaciones y las desaminaciones dependientes de este coenzima (en las rutas de la serina, la cisteína, la metionina y la treonina).

- La Segunda etapa, en el transcurso de la cual tendría lugar el desarrollo de las descarboxilaciones (con recurso a: tiamin-pirofosfato, lipoamida, FAD y coenzima A), y en la que se alcanzaría el desarrollo definitivo del metabolismo correspondiente a los aminoácidos del grupo 3.

- Finalmente, en el desarrollo del metabolismo de los aminoácidos, se puede distinguir una Tercera etapa, durante la cual se completaría el desarrollo definitivo del metabolismo correspondiente al grupo 4 y al ciclo del cítrico.

El análisis cladístico aplicado a las rutas de demolición de los aminoácidos alifáticos confirma la secuencia de etapas que proponemos (Cunchillos y Lecointre, 1999).

La segunda gran fase considerada corresponde, en nuestra opinión, a dos procesos relativamente independientes (el desarrollo del metabolismo correspondiente a los ácidos grasos y a los monosacáridos), que pudieron coincidir en el tiempo. Para sim- 
plificar, vamos a suponer que tuvieron lugar en dos etapas, aunque, insistimos, pudieron ser más o menos simultáneos. Se distinguen así dos nuevas etapas:

- La Cuarta etapa que correspondería al desarrollo de las rutas de síntesis y demolición de los ácidos grasos.

- La Quinta etapa sería la correspondiente al desarrollo del metabolismo de los monosacáridos.
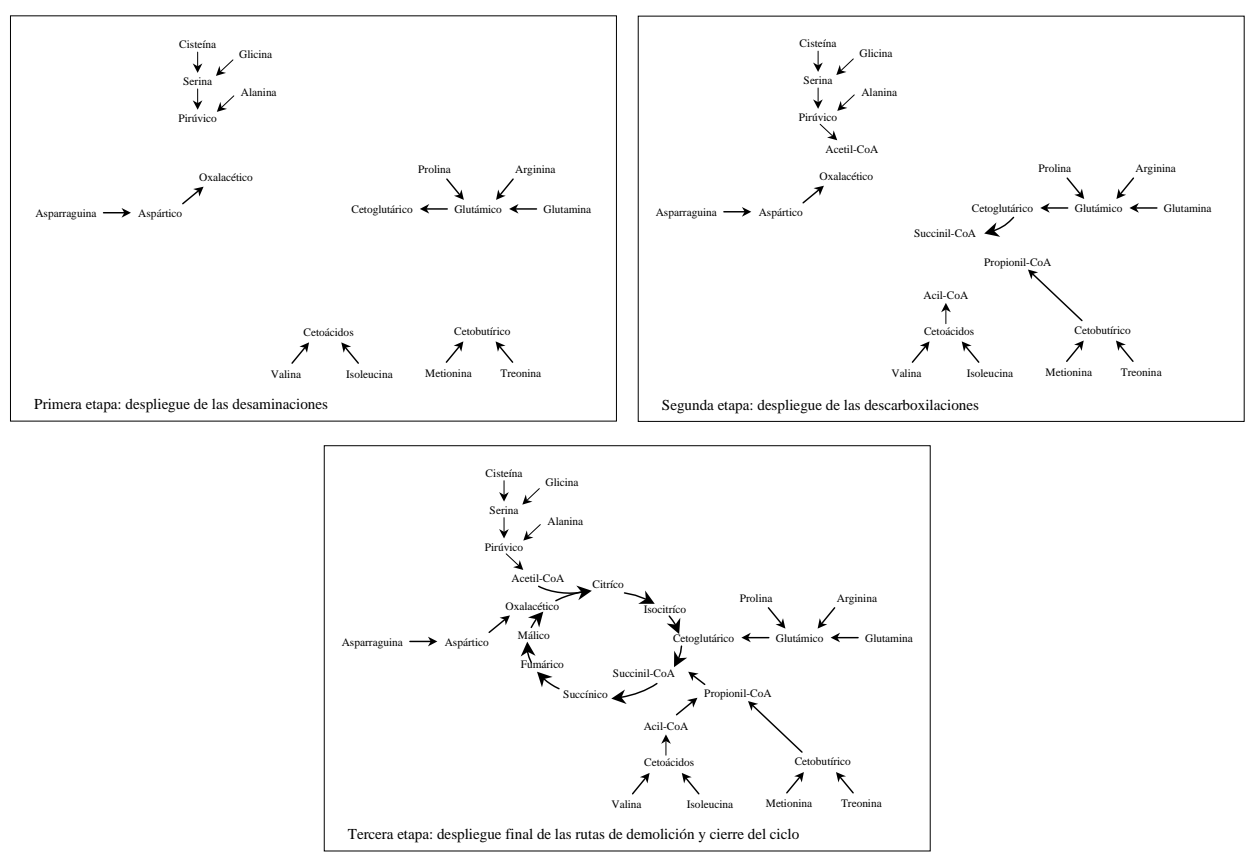

Figura 7.- Etapas propuestas en el desarrollo evolutivo de las rutas de demolición de los aminoácidos alifáticos

Por razones de espacio, nos hemos limitado aquí a presentar las grandes líneas del desarrollo del metabolismo celular. Este esquema se ve plenamente confirmado por el análisis comparado de las transformaciones metabólicas, que permite, además, hacer un desarrollo muy detallado de cada una de las etapas que acabamos de enumerar (Cordón, 1990). 


\section{CHOMIN CUNCHILLOS}

\section{BIBLIOGRAFÍA}

BertalanfFy, L. Von (1976), Teoría general de los sistemas, Madrid, Fondo de cultura económica.

Bunge, M. (1959), Causality. The place of the causal principle in modern science, Cambridge (Mass.), Harvard University Press.

CoRdón, F. (1977), La alimentación, base de la biología evolucionista, Madrid, Alfaguara.

- (1990), Tratado evolucionista de biología, Madrid, Aguilar.

- (1994), «Las proteínas globulares: su estructura y función supramolecular», Mundo científico, 142 , p. 40-47.

- (1994), «Las proteínas globulares: su coordinación en el soma celular y su origen desde una evolución molecular», Mundo científico, 143, p. 144-151.

CunchILlos, Ch. (1997), «Introduction à la théorie des niveaux d'intégration», en TORT P. (dir.), Pour Darwin, Paris, PUF, p. 379-399.

- (1997), «Les grands axes de l'évolution du métabolisme cellulaire», en P. TORT (dir.), Pour Darwin, Paris, PUF, p. 425-447.

Cunchillos, Ch. y LeCoINTRE, G. (1999), «Phylogenetic analysis of metabolic pathways of aliphatic aminoacid degradation», comunicación al XVIIIth meeting of the Willi Hennig Society, Goettingen, 13-17 Sept. 1999.

- (1999), «Analyse phylogénétique des voies de degradation des acides aminés aliphatiques», comunicación al congrès 1999 de la Société Française de Systématique: «caractères», 22-24 Sept. 1999.

Horowitz, N. H. (1945), «On the evolution of biochemical syntheses», Proc. Natl. Acad. Sci., 31, p. 153-157.

Kluyver, A. J. (1926), «Die Einheit in der Biochemie», Chemie der Zelle und Gewebe, 13, p. 134- 190.

KNOWLES, J.R. (1991), «Enzyme catalysis: not different, just better», Nature, 350 / 6314, p. 121-124.

Mayr, E. (1988), Toward a New Philosophy of Biology, Cambridge (Mass.), Harvard University Press.

RosenBerg, A. (1985), The Structure of Biological Science, Cambridge, Cambridge University Press.

TORT, P. (1997), Darwin et le darwinisme, Paris, PUF.

VAN NIEL, C. B. (1949), «The 'Delft school' and the rise of general microbiology», Bacteriological Rewiews, 13, p. 161-174.

ZUCKERKANDL, E. y PAULING, L. (1965), «Molecules as documents of evolutionary history», J. Theoret. Biol., 8, p. 357-366. 